\title{
Impact of Healthcare Information Technology on Nursing Practice
}

\author{
Ronald J. Piscotty, Jr., PhD, RN-BC ${ }^{1}$, Beatrice Kalisch, PhD, RN, FAAN ${ }^{2}$, \& Angel Gracey-Thomas ${ }^{3}$ \\ 1 Theta Psi, Assistant Professor, Wayne State University College of Nursing, Detroit, MI \\ 2 Rho, Titus Professor, University of Michigan School of Nursing, Ann Arbor, MI \\ 3 Theta Psi, Research Assistant, Wayne State University College of Nursing, Detroit, MI
}

Key words

Clinical decision support, missed care, nursing, reminders

\section{Correspondence}

Dr. Ronald J. Piscotty, Jr., Assistant Professor, Wayne State University College of Nursing, Detroit, MI 48202. E-mail: piscotty@wayne.edu

Accepted: March 23, 2015

doi: $10.1111 /$ jnu. 12138

\begin{abstract}
Purpose: To report additional mediation findings from a descriptive cross sectional study to examine if nurses' perceptions of the impact of healthcare information technology on their practice mediates the relationship between electronic nursing care reminder use and missed nursing care.

Design: The study used a descriptive design. The sample $(N=165)$ was composed of registered nurses working on acute care hospital units. The sample was obtained from a large teaching hospital in Southeast Michigan in the fall of 2012. All eligible nursing units $(n=19)$ were included.

Methods: The MISSCARE Survey, Nursing Care Reminders Usage Survey, and the Impact of Healthcare Information Technology Scale were used to collect data to test for mediation. Mediation was tested using the method described by Baron and Kenny. Multiple regression equations were used to analyze the data to determine if mediation occurred between the variables.

Findings: Missed nursing care, the outcome variable, was regressed on the predictor variable, reminder usage, and the mediator variable impact of technology on nursing practice. The impact of healthcare information technology (IHIT) on nursing practice negatively affected missed nursing care $(t=-4.12$, $p<.001$ ), explaining $9.8 \%$ of variance in missed nursing care. With IHIT present, the predictor (reminder usage) was no longer significant $(t=-.70$, $p=.48)$. Thus, the reduced direct association between reminder usage and missed nursing care when IHIT was in the model supported the hypothesis that IHIT was at least one of the mediators in the relationship between reminder usage and missed nursing care.

Conclusions: The perceptions of the impact of healthcare information technology mediates the relationship between nursing care reminder use and missed nursing care. The findings are beneficial to the advancement of healthcare technology in that designers of healthcare information technology systems need to keep in mind that perceptions regarding impacts of the technology will influence usage.

Clinical Relevance: Many times, information technology systems are not designed to match the workflow of nurses. Systems built with redundant or impertinent reminders may be ignored. System designers must study which reminders nurses find most useful and which reminders result in the best quality outcomes.
\end{abstract}

A major challenge facing nurses today is the demand of providing safe and quality care, while still being efficient and cost effective. Implementation of technology in various aspects of our lives continues. The trend in health care is the introduction of technology to improve both quality of care and decreased costs. Thus, finding methods that can help nurses offer safe and effective care using technology is an absolute necessity. In order to achieve 
these ambitious goals, the reduction of healthcare errors is requisite. This includes reducing the occurrence of missed nursing care (required nursing care not delivered or significantly delayed). Missing required nursing care or delaying care contributes to poor patient outcomes (Kalisch \& Xie, 2014). Common themes of missed nursing care include basic nursing care such as ambulation, patient turning, feeding, and bathing (Piscotty $\&$ Kalisch, 2014a).

Technology is being implemented as a tool to prevent healthcare errors. The technology of interest in this study is the use of clinical decision support systems (CDSS). CDSS have long been used by physicians and are now being used by nurses to guide clinical practice (Choi, Choi, Bae, \& Lee, 2011) and to improve patient outcomes (Choi et al., 2011; Piscotty \& Kalisch, 2014b; Staggers, Weir, \& Phansalkar, 2008). Electronic nursing care reminder usage, a type of CDSS, is related to decreased reports of missed care (Piscotty \& Kalisch, 2014c; Piscotty, Kalisch, Gracey-Thomas, \& Yarandi, 2015). Technology is meant to augment nurses' clinical reasoning, not replace it. Combining technology and excellent clinical reasoning will more likely lead to a decrease in errors and an improvement in both quality and safety.

The purpose of this study is to report additional mediation findings from a descriptive cross-sectional study that examined the relationship between missed nursing care and electronic nursing care reminders (Piscotty $\&$ Kalisch, 2014c). The research question examined in this report is: Do nurses' perceptions of the impact of healthcare information technology (IHIT) on their practice mediate the relationship between electronic nursing care reminder use and missed nursing care? It is hypothesized that nurses who have more favorable perceptions of healthcare technology will use the technology more readily (e.g., reminders) and therefore sustain decreased amounts of missed nursing care.

\section{Literature Review}

\section{Electronic Nursing Care Reminders}

Meaningful use of healthcare information technology (HIT) is now a requirement to receive complete reimbursement from both Medicare and Medicaid (HealthIT.gov, n.d.). The objectives of meaningful use include ensuring quality and safety while providing and improving care communication and management (Madison \& Staggers, 2011). Even with the meaningful use requirements, embracing the electronic healthcare record (EHR) as a tool in the delivery of care has been challenging (Bove \& Jesse, 2010). When nurses view documentation as a difficult and cumbersome task, it often slows the technology's acceptance. Alternatively, if the workflow is designed with the nurse in mind, adoption will be increased (Bove $\&$ Jesse, 2010). Several components of the EHR offer advantages in the delivery of complete nursing care. CDSS with nursing care reminders is a specific tool nurses have to provide quality care and is a necessary requirement to attest to meaningful use of HIT.

A review of the current literature did not locate articles that specifically address electronic nursing care reminders. This is a gap in our current understanding of the types of CDSS nurses use, find helpful, or prefer for delivery (Staggers et al., 2008). Choi and colleagues (2011) reported that intensive care unit (ICU) nurses are more likely to adopt CDSS if integration exists with physiologic monitors. Additionally, they reported that necessary documentation of care must be available in the EHR to improve adoption (Choi et al., 2011). The integration of documentation that eliminates the duplication of work is essential for adoption of the EHR (Bove \& Jesse, 2010; Choi et al., 2011). Future research and continuous evaluation of electronic nursing care reminders is necessary in order to ensure accuracy and quality improvement.

\section{Missed Nursing Care}

Basic nursing duties including (but not limited to) feeding, bathing, ambulation, turning, and hygiene are common nursing care activities that are often missed (Kalisch, 2006). While the importance of basic nursing care is taught to nurses in their first year of education, these care items are some of the first to not be completed. Pressure ulcer development and pneumonia are just two complications that can be prevented when basic nursing care is delivered in a timely fashion. These complications may result in decreased quality of life for patients and increased healthcare costs. Reasons nurses do not complete these activities have been found to be related to a lack of staffing and material resources and a decrease in communication with fellow staff and patients (Kalisch, Landstrom, \& Williams, 2009).

Three studies have examined solutions in order to reduce the omission of nursing care and improve quality of care (Kalisch, Xie, \& Ronis, 2013; Piscotty \& Kalisch, 2014c; Piscotty et al., 2015). Kalisch and colleagues (2013) reported that successful teamwork on a unit is significantly related to decreased reports of missed nursing care. Nursing care reminders embedded in the EHR have been found to be related to a decrease in the occurrence of missed nursing care (Piscotty \& Kalisch, 2014c; Piscotty et al., 2015). In the studies conducted by Piscotty, it was identified that nurses who utilize the electronic nursing care reminders more frequently report 


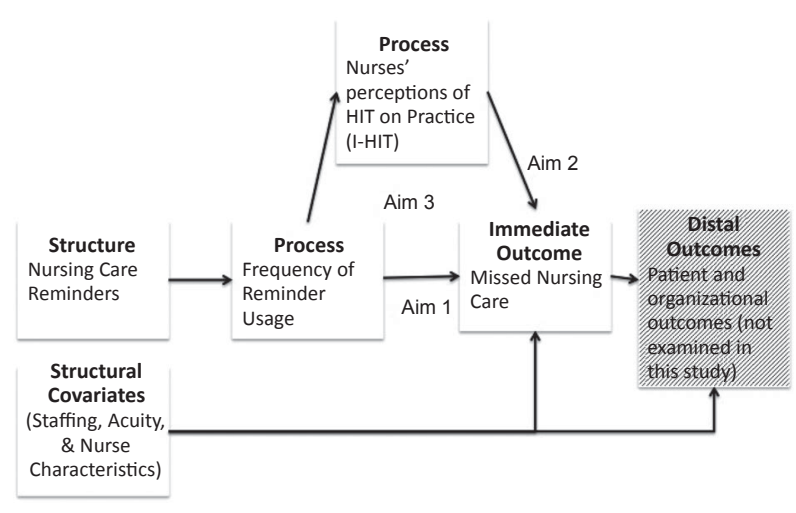

Figure 1. Modified structure process outcome model.

less missed care (Piscotty \& Kalisch, 2014c; Piscotty et al., 2015).

\section{Conceptual Framework}

The conceptual framework chosen for this study is the Structure, Process, and Outcomes Model of Healthcare Quality (Donabedian, 2005). This framework can be used to understand the relationships that nursing care reminders (structure) and their use (process) have on missed nursing care (immediate outcome) and patient and organizational outcomes (distal outcomes; Figure 1). The examination of distal outcomes is beyond the scope of this study and will not be explored. Additionally, a process-mediating variable of nurses' perceptions of the impact of HIT on practice (IHIT) is included in the model. Conceptual definitions and empirical indicators for each variable are listed in Table $\mathbf{1}$.

\section{Methods}

The sample, design, measures, and procedures are described in detail in our previous publication (Piscotty $\delta$ Kalisch, 2014c). A brief summary of methods will be presented here.

\section{Design, Sample, and Setting}

The main study utilized a descriptive cross-sectional design with a convenience sample (Piscotty \& Kalisch, $2014 \mathrm{c})$. The sample $(\mathrm{N}=165)$ consisted of registered nurses (RNs) working on acute care hospital units in a large teaching hospital in Southeast Michigan in 2012. All eligible nursing units $(\mathrm{n}=19)$ were included in the study. Inclusion criteria included that participants had to be RNs that took a daily patient assignment, the EHR had to be implemented for at least 6 months, and the EHR had to include the types of reminders that were examined in the study. Exclusion criteria included non-RN employees (nursing assistants, clerks, patient care associates, nursing students, etc.) and RNs that did not take a daily patient assignment (managers, educators, nursing instructors, case managers, etc.).

\section{Measures}

Nursing Care Reminder Usage Survey (NCRS). The NCRS was used to measure frequency of reminder use in this study. The investigators of this study developed the NCRS measurement tool. The survey contains 12 questions regarding usage of nursing care reminders (see Table 2). The following definition regarding nursing care reminders was included in the survey directions: A nursing reminder is an electronic list, prompt, or cue of tasks or procedures that need to be completed by either the nurse or nursing attendant during the shift. Therefore, all questions are asked in the context of electronic reminders. Additional information including instrument validity and reliability has been published elsewhere (Piscotty \& Kalisch, 2014c).

Impact of Health Information Technology (IHIT) Scale. The I-HIT Scale was used to measure nurse perceptions about the impact of HIT on practice (Dykes, Hurley, Cashen, Bakken, \& Duffy (2007). The I-HIT Scale is composed of 29 items contained in four subscales (Dykes et al., 2007). Additional information, including instrument validity and reliability, has been published elsewhere (Dykes et al., 2007; Piscotty \& Kalisch, 2014c).

Missed Nursing Care. The Missed Nursing Care Survey (MISSCARE Survey) is a two-part survey and a demographics section that measures the extent to which elements of nursing care are missed as well as the reasons for missing care (Kalisch \& Williams, 2009). Part A of the survey and a demographics section were used in the study to measure elements of missed nursing care (Piscotty \& Kalisch, 2014c; Piscotty et al., 2015). Instrument validity and reliability has been published elsewhere (Kalisch \& Williams, 2009).

\section{Procedures}

Institutional review board approval was obtained prior to the study. Online surveys were used, with links to the surveys sent to each participant via e-mail. Detailed instructions, consent information, and links to the study instruments were included. The surveys were administered using the Qualtrics (Provo, UT, USA) survey software. The surveys were anonymous and no identifying information was collected. Respondent burden was 
Table 1. Conceptual Definitions and Empirical Indicators: Dependent, Independent, and Mediating Variables

\begin{tabular}{|c|c|c|}
\hline Dependent variable & Conceptual definition & Empirical indicators \\
\hline Missed nursing care & $\begin{array}{l}\text { Kalisch, Landstrom, \& Hinshaw (2009) defined missed } \\
\text { nursing care in a concept analysis. Missed nursing care } \\
\text { is defined as any aspect of required patient care that is } \\
\text { omitted (either in part or whole) or delayed. }\end{array}$ & $\begin{array}{l}\text { Missed nursing care is defined } \\
\text { operationally as: The total score on } \\
\text { the MISSCARE survey (Kalisch \& } \\
\text { Williams, 2009). }\end{array}$ \\
\hline Independent variable & Conceptual definition & Empirical indicators \\
\hline $\begin{array}{l}\text { Level of use of EHR } \\
\text { nursing care } \\
\text { reminders }\end{array}$ & $\begin{array}{l}\text { The registered nurses' self-rated level of use of nursing } \\
\text { care reminders in their facilities' EHR. }\end{array}$ & $\begin{array}{l}\text { Level of use of nursing care reminders } \\
\text { is operationally defined as the } \\
\text { nurse's total score on the nursing } \\
\text { care reminders survey. }\end{array}$ \\
\hline Mediating variables & Conceptual definition & Empirical indicators \\
\hline $\begin{array}{l}\text { Impact of healthcare } \\
\text { information } \\
\text { technology on } \\
\text { nursing practice }\end{array}$ & $\begin{array}{l}\text { Nurses' perceptions of the influence that HIT has on } \\
\text { interdisciplinary communication, workflow patterns, } \\
\text { and satisfaction with HIT applications available in } \\
\text { hospitals. }\end{array}$ & $\begin{array}{l}\text { Total score on the I-HIT Scale (Dykes } \\
\text { et al., 2007). }\end{array}$ \\
\hline
\end{tabular}

Table 2. Nursing Care Reminders Survey

How frequently do you utilize the following types of nursing care reminders to assist you in completing nursing care activities?

1. A paper list of reminders based on what is in the electronic healthcare record (EHR)

2. Printout of list of care activities that serve as a reminder

3. Electronic nursing care orders that serve as a reminder

4. List of nursing care activities in plan of care that serve as a reminder

5. Electronic list of reminders (i.e., task list, documentation checklist, documentation form, work queue, work list)

6. Electronic list of reminders not in the EHR

7. Computerized provider order entry (CPOE) list that serves as a reminder

8. Electronic documentation in the EHR that serves as a reminder

9. Electronic checklist for documenting care that serves as a reminder

10. Alert of reminder message pop-ups in the EHR

11. How frequently do you utilize nursing care reminders to assist you in completing nursing care activities?

12. How helpful do you find the electronic nursing care reminders?

considered to be minimal as the instruments were short and each took less than 10 min to complete. Nurses were reminded via flyers placed in high-visibility areas on the units. In addition, reminder e-mail messages were sent to all nurses twice a week. Surveys were collected within 1 month from the start of the study.

\section{Data Analyses}

Data were analyzed using SPSS 21 (IBM Corp., Armonk, NY, USA). Data were initially examined through descriptive analysis, and total scores were calculated for each of the three main variables in the study. Assumptions for multiple linear regressions were assessed. Missing data were excluded casewise for analysis. The alpha level for all analyses was set at .05 or less.

In order to test for mediation, the method described by Baron and Kenny (1986) was used, in which a variable is considered a mediator (Figure 2) when three criteria are met: (a) variation in the independent variable (reminder usage) accounts for significant variation in the

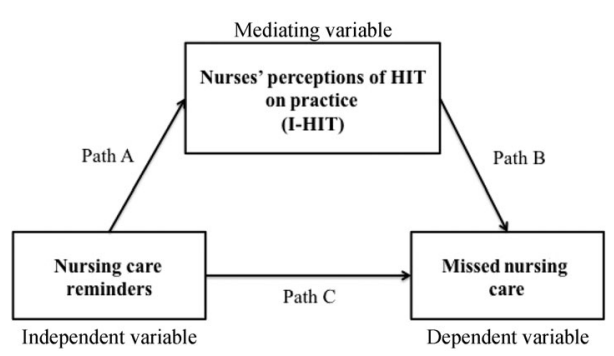

Figure 2. General mediation model.

mediator variable (I-HIT Scale; path a), (b) variation in the mediator variable (I-HIT Scale) accounts for significant variation in the dependent variable (missed care; path b), and (c) when paths a and b are controlled, there is significant reduction in the variance between the independent variable (reminder usage) and dependent variable (missed care; path c). When these three criteria are met, the relationship between the independent variable (reminder usage) and the dependent variable (missed 
care) must be less in the third equation than in the second (Baron \& Kenny, 1986).

\section{Results}

\section{Sample}

The sample $(N=165)$ consisted of staff nurses employed at a large teaching hospital in Southeast Michigan. Respondents $(69.1 \%, n=114)$ primarily held a baccalaureate degree as their highest level of education, with $66.7 \%(n=110)$ of those participants having a bachelor of science degree in nursing (BSN; Piscotty $\delta$ Kalisch, 2014c). The majority of respondents were female $(87.9 \%, n=145)$ and between the ages of 25 and 34 years $(37.0 \%, n=61$; Piscotty $\&$ Kalisch, 2014c). The majority of the respondents worked full-time $(93.3 \%$, $n=154)$, and over half of the participants in the study $(63.0 \%, n=104)$ worked on a medical surgical unit (Piscotty \& Kalisch, 2014 c).

\section{Surveys}

Missed nursing care, reminder usage, I-HIT Scale (descriptives). Total missed nursing care scores ranged from a low of 24 to a maximum of $84(M=56.09$; $S D=11.79$ ) out of a total possible score of 120. Nursing care reminders total scores ranged from a low of 11 to a maximum of $50(M=29.98 ; S D=8.11)$ out of a total possible score of 60 . Total I-HIT Scale scores ranged from 28 to $171(M=129.32 ; S D=22.94)$ out of a total possible score of 174 .

I-HIT scale mediation of the effect of reminder usage on missed nursing care. The IHIT was hypothesized in this study as a mediating variable in the relationship between nursing care reminders (NCRS) and missed nursing care. To satisfy the requirements for mediation, three regression equations were computed. To establish mediation, the following conditions had to be satisfied: (a) NCRS must affect IHIT; (b) NCRS must affect missed nursing care in the second equation; and (c) IHIT must affect missed nursing care in the third equation. A strong demonstration of mediation occurs when the relationship between the NCRS and IHIT is not significant (Krause et al., 2010).

In Equation 1, the I-HIT Scale, the mediator variable, was regressed on the predictor variable, the NCRS. As noted in Figure 3, results indicated that the NCRS was significantly associated with IHIT $\left(F_{156}=19.84, p<.001\right)$. The NCRS explained $11.3 \%$ of the variance in the IHIT scores.

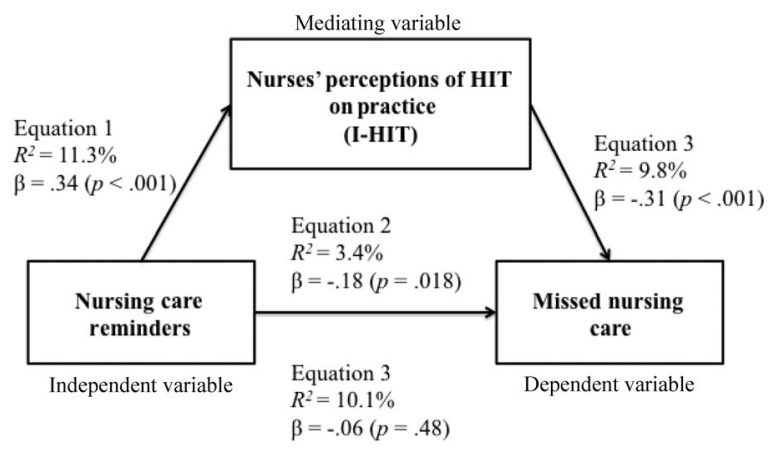

Figure 3. Test of the mediation model with regression analyses.

In Equation 2 , missed nursing care, the outcome variable, was regressed on the predictor variable, the NCRS. The NCRS was significantly associated with missed nursing care $\left(F_{163}=5.67, p=.018\right)$. The NCRS explained $3.4 \%$ of the variance in missed nursing care.

In Equation 3, missed nursing care, the outcome variable, was regressed on the predictor variable, the NCRS, and the mediator variable (IHIT). IHIT negatively affected missed nursing care $(t=-4.12, p<.001)$, explaining $9.8 \%$ of variance in missed nursing care. With IHIT present, the predictor (NCRS) was no longer significant $(t=-.70, p=.48)$. Thus, the reduced direct association between the NCRS and missed nursing care when IHIT was in the model supported the hypothesis that IHIT was at least one of the mediators in the relationship between the NCRS and missed nursing care.

\section{Discussion}

Analysis of the mediation results supports the research question that perceptions of the influence of HIT mediates the relationship between reminder use and missed nursing care. Nurses who use the electronic reminders more frequently and have higher perceptions about the impact of HIT on their practice have less missed nursing care than nurses who use the reminders but have neutral or negative perceptions of the impact of HIT.

This is a significant finding because nurses who have more positive perceptions of the impact of HIT on their practice have less missed nursing care than nurses who use the reminders without positive perceptions of their value. This is an important consideration since healthcare organizations can utilize the I-HIT Scale to assess whether or not their nurses have positive perceptions about the technology systems they are required to use. Organizations can then target specific system design or workflow changes to improve nurses' perceptions of the impact of HIT on their practice. 
Although the mediating relationship between missed nursing care, perceptions of the impact of HIT, and nursing care reminders had not been previously studied, Dykes et al. (2007) hypothesized that nurses who have positive perceptions of the impact of HIT on their practice would be more likely to use the technology. This hypothesis is supported by the findings from this study. Our findings are similar to previous studies that found that CDSS must be aligned with the nurses' workflow if they are to use the information systems effectively (Choi et al., 2011; Courtney, Alexander, \& Demiris, 2008; Piscotty \& Kalisch, 2014b; Piscotty \& Tzeng, 2011 ). Saleem et al. (2005) reported that one facilitator to using CDSS by nurses was to integrate the reminders into the nurses' daily clinical workflow.

An alternate explanation for this finding is that nurses who use nursing care reminders already have more positive perceptions of the impacts of HIT on their practice. Nurses who utilize the system may be more accountable and therefore have decreased amounts of missed nursing care to begin with. Organizational or cultural factors may also influence nurses' perceptions of the impact of HIT on their practice.

\section{Limitations}

Limitations included threats to internal and external validity. These threats were addressed through a priori power analysis, using established instruments, and collecting data on multiple nursing units.

\section{Conclusions}

Our study supports that perceptions of the impact of HIT mediates the relationship between nursing care reminder use and missed nursing care. The findings are beneficial to the advancement of healthcare technology in that designers of HIT systems need to keep in mind that perceptions regarding the impact of the technology will influence usage. Many times, information technology systems are not designed to match the workflow of nurses. Systems built with redundant or impertinent reminders may be ignored. System designers must study which reminders nurses find most useful and which reminders result in the best quality outcomes.

\section{Clinical Resources}

- The Agency for Healthcare Research and Quality (AHRQ) Patient Safety Network (PSNet) is a national web-based resource featuring the latest news and essential resources on patient safety: http://psnet.ahrq.gov
- The Institute for Healthcare Improvement (IHI) is an independent not-for-profit organization and is a leading innovator, convener, partner, and driver of results in health and healthcare improvement worldwide: http://www.ihi.org

\section{References}

Baron, R., \& Kenny, D. (1986). The moderator-mediator variable distinction in social psychological research: Conceptual, strategic, and statistical considerations. Journal of Personality and Social Psychology, 51(6), 1173-1182.

Bove, L., \& Jesse, H. (2010). Worklists: Helping to transform nursing care. ANIA-CARING Newsletter, 25(1), 1-7.

Choi, M., Choi, R., Bae, Y., \& Lee, S. (2011). Clinical decision support systems for patient safety: A focus group needs assessment with Korean ICU nurses. Computers, Informatics, Nursing, 29(11), 671-678.

Courtney, K., Alexander, G., \& Demiris, G. (2008). Information technology from novice to expert: Implementation implications. Journal of Nursing Management, 16(6), 692-699.

Donabedian, A. (2005). Evaluating the quality of medical care. Milbank Memorial Fund Quarterly, 83(4), 691729, (reprinted from Milbank Memorial Fund Quarterly, 44(3), 166-203).

Dykes, P. C., Hurley, A., Cashen, M., Bakken, S., \& Duffy, M. E. (2007). Development and psychometric evaluation of the Impact of Health Information Technology (I-HIT) Scale. Journal of the American Medical Informatics Association, 14(4), 507-514.

HealthIT.gov. (n.d.). EHR incentives $\theta$ certification: Meaningful use definition and objectives. Retrieved from http://www.healthit.gov/providers-professionals/ meaningful-use-definition-objectives.

Kalisch, B. (2006). Missed nursing care: A qualitative study. Journal of Nursing Care Quality, 21(4), 306-313.

Kalisch, B., \& Xie, B. (2014). Errors of omission: Missed nursing care. Western Journal of Nursing Research, 36(7), 875-890.

Kalisch, B., Xie, B., \& Ronis, D. L. (2013). Train-the-trainer intervention to increase nursing teamwork and decrease missed nursing care in acute care patient units. Nursing Research, 62(6), 405-413.

Kalisch, B. J., Landstrom, G. L., \& Hinshaw, A. S. (2009). Missed nursing care: A concept analysis. Journal of Advanced Nursing, 65(7), 1509-1517.

Kalisch, B. J., Landstrom, G., \& Williams, R. A. (2009). Missed nursing care: Errors of omission. Nursing Outlook, 57(1), 3-9.

Kalisch, B. J., \& Williams, R. A. (2009). Development and psychometric testing of a tool to measure missed nursing care. Journal of Nursing Administration, 39(5), 211-219. 
Krause, M., Serlin, R., Ward, S., Rony, R., Ezenwa, M., \& Naab, F. (2010). Testing mediation in nursing research: Beyond Baron and Kenny. Nursing Research, 59(4), 288-294.

Madison, M., \& Staggers, N. (2011). Electronic health records and the implications for nursing practice. Journal of Nursing Regulation, 1(4), 54-60.

Piscotty, R., \& Kalisch, B. (2014a). Lost opportunities ... the challenges of "missed nursing care." Nursing Management, 45(10), 40-44.

Piscotty, R., \& Kalisch, B. (2014b). Nurses' use of clinical decision support: A literature review. Computers, Informatics, Nursing, 32(12), 562-568.

Piscotty, R., \& Kalisch, B. (2014c). The relationship between electronic nursing care reminders and missed nursing care. Computers, Informatics, Nursing, 32(10), $475-481$.
Piscotty, R., Kalisch, B., Gracey-Thomas, A., \& Yarandi, H. (2015). Electronic nursing care reminders: Implications for nurse leaders. Journal of Nursing Administration.

Piscotty, R., \& Tzeng, H. (2011). Exploring the clinical information system implementation readiness activities to support nursing in hospital settings. Computers, Informatics, Nursing, 29(11), 648-656.

Saleem, J., Patterson, E., Militello, L., Render, M., Orshansky, G., \& Asch, S. (2005). Exploring barriers and facilitators to the use of computerized clinical reminders. Journal of the American Medical Informatics Association, 12(4), 438-447.

Staggers, N., Weir, C., \& Phansalkar, S. (2008). Patient safety and health information technology: Role of the electronic health record. In R. G. Hughes (Ed.), Patient safety and quality: An evidenced-based handbook for nurses (pp. 1234-1276). AHRQ Publication No. 08-0043. Rockville, MD: Agency for Healthcare Research and Quality. 